\title{
The Challenge of Vaccine Nationalism
}

\author{
Keltie Hamilton $^{1}$, Devanshi Shah ${ }^{1}$ and Danica Fitzsimmons ${ }^{2}$ \\ ${ }^{1}$ Public Health Sciences, Dalla Lana School of Public Health, University of Toronto, Toronto, Canada \\ 2 Public Health, Health Sciences, University of Lethbridge, Lethbridge, Canada \\ Corresponding author: Keltie Hamilton, keltie.hamilton@mail.utoronto.ca
}

\section{Abstract}

The COVID-19 pandemic has had a devastating impact on global health for almost two years, resulting in nearly 200 million cases and over 4 million deaths worldwide. Despite a range of non-invasive public health measures, (i.e. physical distancing, and masks) vaccines have been one of the more critical and effective interventions to slow the pandemic. Produced at record-breaking speeds, the highly efficacious mRNA vaccines represented hope for many. Including global health organizations who have called for strategies to maximize vaccine equity since their conception. While many high-income countries (HICs) agreed to prioritize global vaccine equity; in truth, individual health outweighed community health. The reality of HICs vaccine purchasing behaviors and distribution have exposed a different agenda - one that aligns with a neoliberal emphasis on individuals and profits at the expense of global good. This commentary questions the efficacy of global health agreements and the commitment from wealthy countries to address global health inequities through a one health framework. Ultimately, concluding that the path to global vaccine equity will require a commitment to global good. Vaccine nationalism and lack of equitable global health policy continues to fuel a never-ending health crisis. HICs must be held accountable for the lack of commitment to global health equity.

Keywords: Global Health, Public Health, Public Health Policy, One Health, Health Equity, COVID-19, Coronavirus, Pandemic, Health Politics

In November 2019, SARS-CoV-2, the virus that causes COVID-19 disease (hereafter COVID-19), emerged from Wuhan, China. ${ }^{1}$ Following health expert recommendation, the World Health Organization (WHO) announced travel precautions by January $9^{\text {th }}, 2020 .^{2}$ Shortly after the WHO declared COVID-19 as a global pandemic on March $11^{\text {th }} 2020 .^{3}$ Novel and highly infectious, COVID-19 quickly spread through the global population. Some groups experienced a far greater burden of severe morbidity (e.g., stroke, severe respiratory infections, organ failure) and risk of premature mortality. ${ }^{4}$ Severe viral infections like COVID-19 require complex medical care and resources to prevent death and other adverse health outcomes. These resources are in short supply for much of the world. Given COVID-19's high risk for morbidity and mortality, as well as the social and economic challenges associated with lockdowns, the development of an effective vaccine (alongside other public health measures [i.e., social distancing, masks]) was paramount. At 
record-breaking speeds, a number of pharmaceutical companies developed several candidate vaccines, with the most effective utilizing messenger ribonucleic acid (mRNA) technology.

While the mRNA technology has been around for decades, primarily for cancer research, it has never been mass-produced on a global scale before. ${ }^{5}$ The pace at which COVID-19 vaccines have been created, tested, and approved has never been accomplished in human history. It would have been impossible without collaborations among wealthy donors, academic institutions, and health authorities. This allowed for the rapid funding and approval of COVID-19 vaccines for use.

To encourage equitable access to vaccines, WHO created the COVID-19 Vaccines Global Access (COVAX) group. The goal of COVAX is to achieve worldwide vaccination - of which 170 countries cosigned. ${ }^{6}$ Equity versus equality is a consistent and underlying theme in the fight for global health. This is because we live in a world where access to money and living in wealthier countries improves health outcomes. Equity refers to allocating resources based on individual circumstances and needs, whereas equality refers to allocating equal resources to all. ${ }^{7}$ Critically, an equity perspective acknowledges that to achieve health equity, some individuals or populations may need a different amount or type of resource relative to others. True vaccine equity would require a strategy that prioritizes populations most at risk of severe COVID-19 regardless of their country's financial status. ${ }^{8}$ Despite this objective and shared global value, realworld vaccine distribution has largely favored high-income nations. This has been at the expense of those living in countries with limited health care resources.

This commentary situates current issues and challenges with COVID-19 vaccine equity to an increased neoliberal emphasis on individuals, limited regulation, free trade and maximizing monetary gains. Ultimately, the commodification and subsequent expense of healthcare widens the health and economic divide between lowmiddle income countries (LMIC) and highincome countries (HIC). This causes harm to those without the financial capital to compete for access to necessary healthcare commodities, such as vaccines.

Equity in health is something the world struggled with long before the COVID-19 pandemic. However, over the past 18 months, the health inequalities present between HIC and LMIC have been at the forefront of pandemic response. Gaps in our willingness to enact protective public health policy on a global scale were highlighted when COVID-19 was identified as a worldwide threat to health. Despite calls to action from international organizations, such as the WHO and United Nations (UN), there was no cohesive global plan or response to COVID-19. In addition to the virus itself, the lack of global coordination and policy regarding pandemic responsiveness represented another major threat to health. In defiance of calls from the WHO and UN to makes vaccines accessible and affordable, little was accomplished on the global scale. These early cracks in our 
pandemic response have grown under the immense strain of the pandemic and are resulting in major global disparities in vaccine access.

Gaps in the reach and influence of global health organizations have had an enduring and negative impact on the global community's health. ${ }^{8}$ Although the UN and WHO have the support of 193 member states, ${ }^{9}$ many have directly challenged or ignored their expert recommendations and sought to prioritize their citizens by securing premarket vaccines in quantities that clearly ignore the global good. By prioritizing singular nations for initial vaccination allocation, countries have shifted away from the goal of global vaccine access to an individualistic ideology, commonly referred to as vaccine nationalism. This shift reflects a change from the values and priorities present in prior global health crises, such as the eradication of smallpox, where the individual countries prioritized the global good over profit. ${ }^{10}$ For example, when the inventor of the Polio vaccine, Jonas Salk, was asked about its patent in 1955 he stated, "There is no patent. Could you patent the sun?". 6 Since the development of a COVID-19 vaccine, public health professionals have called for vaccine equity in both cost and allocation ${ }^{11}$ to allow every person on Earth to gain access to this potentially lifesaving vaccination, but this has not been achieved. ${ }^{12}$

Within the COVAX documentation, it is crucial to note the phrasing of " $a$ call for vaccine equity". It is important to note this is largely because the WHO has limited control over vaccine production and allocation. Although this "call" stands to improve global health, without enforceable global policy protection for countries with less economic capital (and regulation of larger, wealthier countries) there is nothing tangible to enact them. The lack of enforceable global health laws and regulations has permitted a flawed global health system to maintain systemic injustices to those already at a disadvantage. This results in countries with economic power prioritizing their citizens over the global good. ${ }^{13}$ Premarket vaccine sales has made this evident, as only wealthy countries with social and political capital were able to secure vaccines. $^{4}$

The residual perspectives of individual health versus collective health are familiar perspectives to many healthcare and public health professionals. Individual health refers to the absence of disease or illness on an individual level and collective health refers to the health of entire populations and the way environmental, social and economic differences impact the health of communities. ${ }^{14}$ However, the ideologies and theories regarding public health interventions that are discussed in lecture halls, at dinner tables, and in board rooms rarely translate perfectly to real-world scenarios. When asked to commit to sharing vaccine technology and vaccination doses, countries avoided the ideals and values rooted in collective health that they reportedly endorse. Instead, they chose a profit-based vaccine approach. This lead to the juxtaposition of having over 170 countries on the COVAX agreement, ${ }^{6}$ yet the majority of the COVID-19 vaccine supply being purchased by HIC before it was even on the 
market. High-income countries like the United States, which feature unfettered capitalism fueled by a strong neoliberal emphasis on deregulation, free trade, individual sovereignty and profit maximization, ${ }^{15}$ were able to purchase and produce massive amounts of vaccine doses. Due to the United States' purchasing power for vaccines, along with major pharmaceutical companies producing vaccines within their borders, their underlying neoliberal political and economic system has naturally bled into global vaccine distribution. This is because the United States and HIC like it are driven to maximize the economic fertility of their markets, subsequently setting the moral tone for vaccine equity and distribution - one that prioritizes profits and an individual country's economic prowess over global health. The moral tone of neoliberalism is one that systemically neglects those living in LMIC due to the lack of financial return, which disproportionately results in increased suffering and mortality for most of the world. The prioritization of individual over collective health is evident due to the prevalence of HIC prioritizing individual health over global good.

The COVAX agreement stated that countries that are self-funded would be guaranteed sufficient vaccinations for the most vulnerable portions of their populations, such as older adults and essential workers. Conversely, countries that need financial support would be guaranteed $20 \%$ of their population to be vaccinated and allocation was to be spread across countries based on dose availability. It is estimated that premarket agreements made with HIC such as the United Kingdom, countries within the
European Union, and the United States resulted in over $50 \%$ of the global vaccine supply being purchased for their citizens despite being only $13 \%$ of the global population. ${ }^{16}$ Canada alone purchased 9.6 COVID-19 vaccine doses per citizen. ${ }^{17}$ This is the highest number of vaccines per citizen globally, with the United Kingdom being the closest per citizen purchase at 5.5 COVID-19 vaccines per citizen. ${ }^{17}$ This inequitable distribution of vaccines has arguably led to variants of concern (VOC) emerging from under-vaccinated populations, such as the Delta variant from India and Lambda variant from Peru. ${ }^{18}$ Until the globe can be vaccinated, areas with inadequate vaccine access will continue to spread COVID-19, increasing the rate of VOCs and endangering every global citizen (even those living in HICs).

The development of variants in LMICs are inevitable without committing to global health as a planetary concern and striving to commit to a one-health approach. One health refers to the concept of focusing on the consequences and responses from interactions between animals, humans and the ecosystem with a particular emphasis on the large burden of disease in LMIC due to resource-poor settings. ${ }^{19}$ Citizens of HIC need to not only commit to a one health approach but also hold their leaders, governments and education systems accountable for improving global health equity in tangible ways. ${ }^{20}$ Without accountability from individual countries, citizens, and policy, the goal of global health equity will never be met. 


\section{Conclusion}

Vaccine nationalism and a lack of global health policy to protect those most at risk calls to question not only vaccination equity, but the ever-widening economic and health divide between HIC and LMIC. The lack of a coordinated, global response to pandemics and limited tangible mechanisms for accountability hampers a community-based and equity-driven approach to vaccine delivery. The dominating neoliberal economies of the United States, Canada and the UK prioritizes their citizens by taking an overly individualistic and markets-driven approach, at the expense of the global majority. Strategies to enforce global health policy and hold HICs accountable for failing to promote global health equity will be essential to ensuring the health of those living in areas without similar access to health and social resources. The promise of a global health equity approach to vaccination need to be fulfilled by HIC, limiting the proliferation of VOCs and lowering COVID cases worldwide.

\section{Acknowledgements}

Thank you to Daniel Harris and Rebecca Christensen for the time and energy spent editing and reviewing this article.

\section{References}

1. Wu F, Zhao S, Yu B, et al. A new coronavirus associated with human respiratory disease in China. Nature. 2020;579(7798):265-269. doi:10.1038/s41586-0202008-3

2. Staff A. A Timeline of COVID-19 Developments in 2020. AJMC. Accessed July 24, 2021.

https://www.ajmc.com/view/a-timeline-of-covid19-developments-in-2020
3. WHO declares novel coronavirus disease a pandemic National | Globalnews.ca. Global News. Accessed July 21, 2021. https://globalnews.ca/news/6660863/whocoronavirus-pandemic/

4. Blumenthal D, Fowler EJ, Abrams M, Collins SR. Covid-19 - Implications for the Health Care System. N Engl J Med. 2020;383(15):1483-1488. doi:10.1056/NEJMsb2021088

5. Jackson NAC, Kester KE, Casimiro D, Gurunathan S, DeRosa F. The promise of mRNA vaccines: a biotech and industrial perspective. Npj Vaccines. 2020;5(1):1-6. doi:10.1038/s41541-020-0159-8

6. Covid-19 vaccine equity as a global good. The BMJ. Published June 25, 2021. Accessed July 9, 2021. https://blogs.bmj.com/bmj/2021/06/25/covid-19-vaccine-equity-as-a-global-good/

7. Linares-Péreza N, Oliva López-Arellano. Health Equity: Conceptual Models, Essential Aspects and the Perspective of Collective Health. Soc Med. 2008;3(3):194206.

8. Weintraub R, Bitton A, Rosenberg ML. The Danger of Vaccine Nationalism. Harv Bus Rev. Published online May 22, 2020. Accessed July 13, 2021. https://hbr.org/2020/05/the-danger-of-vaccine-nationalism

9. Nations U. Member States. United Nations. Published 2021. Accessed July 30, 2021.

https://www.un.org/en/about-us/member-states

10. Barrett S. The Smallpox Eradication Game. Public Choice. 2007;130(1):179-207. doi:10.1007/s11127-0069079-z

11.Emanuel EJ, Persad G, Kern A, et al. An ethical framework for global vaccine allocation. Science. 2020;369(6509):1309-1312. doi:10.1126/science.abe 2803

12. Hall S, Zulu Holt T, Kaplow L, Sunny Sun Y. COVID19 vaccine rollout in LMIC | McKinsey. Published April 2021. Accessed July 9, 2021. https://www.mckinsey.com/industries/healthcare-systems-and-services/our-insights/none-are-safe-until-all-are-safecovid-19-vaccine-rollout-in-low-and-middle-incomecountries

13. Czyzewski K. Colonialism as a Broader Social Determinant of Health. Int Indig Policy J. 2011;2(1):n/a. doi:http://dx.doi.org.myaccess.library.utoronto.ca/10.18584/iipj.2011.2.1.5

14. Swiffen* A. The One vs the Many: When Public Health Conflicts with Individual Rights. Centre for Constitutional Studies. Published May 14, 2020. Accessed July 24, 2021. https://www.constitutionalstudies.ca/2020/05/the-one-vs-the-many-when-publichealth-conflicts-with-individual-rights/

15. Harvey D. A Brief History of Neoliberalism. 1st ed. Oxford University Press; 2007.

16. So AD, Woo J. Reserving coronavirus disease 2019 vaccines for global access: cross sectional analysis. BMJ. 2020;371:m4750. doi:10.1136/bmj.m4750 
17. Kirk A, Sheehy F, Levett C. Canada and UK among countries with most vaccine doses ordered per person. the Guardian. http://www.theguardian.com/world/2021/jan/29/canada-and-uk-amongcountries-with-most-vaccine-doses-ordered-per-person. Published January 29, 2021. Accessed July 30, 2021.

18. The Lancet. COVID-19 in Latin America-emergency and opportunity. Lancet Lond Engl. 2021;398(10295):93. doi:10.1016/S01406736(21)01551-8

19. Mackenzie JS, Jeggo M. The One Health ApproachWhy Is It So Important? Trop Med Infect Dis. 2019;4(2):88. doi:10.3390/tropicalmed4020088

20. Haldane V, Reed AC, Toccalino D, Shan Y, Berry I, Sue-Chue-Lam C. A call for mandatory planetary health education in public health and health services research programs. Univ Tor J Public Health. 2021;2(1). doi:10.33137/utjph.v2i1.34761 